13

\title{
СВЧ-импеданс тонкопленочных гибридных структур сверхпроводник-нормальный металл с большим отношением проводимостей
}

\author{
(C) С.С. Уставщиков ${ }^{1,2}$, А.Ю. Аладышкин ${ }^{1,2}$, В.В. Курин ${ }^{1,2}$, В.А. Маркелов ${ }^{1}$, А.И. Елькина ${ }^{1}$, \\ А.М. Клушин ${ }^{1}$, П.А. Юнин ${ }^{1,2}$, В.В. Рогов ${ }^{1}$, Д.Ю. Водолазов ${ }^{1}$ \\ ${ }^{1}$ Институт фризики микроструктур РАН — фрилиал Института прикладной фризики РАН, \\ Нижний Новгород, Россия \\ ${ }^{2}$ Нижегородский государственный университет им. Н.И. Лобачевского, \\ Нижний Новгород, Россия \\ E-mail: sergey@ipmras.ru
}

Поступила в Редакцию 15 апреля 2019 г.

В окончательной редакции 22 апреля 2019 г.

Принята к публикации 24 апреля 2019 г.

\begin{abstract}
Теоретически и экспериментально исследована температурная зависимость линейного электродинамического отклика тонкопленочных гибридных структур сверхпроводник $(\mathrm{MoN})$ - нормальный металл $(\mathrm{Al})$ с большой разностью проводимостей в нормальном состоянии. Низкочастотные измерения коэффициента взаимной индукции двух катушек с помещенным между ними образцом свидетельствуют об увеличении магнитной экранировки структур сверхпроводник-нормальный металл $(\mathrm{SN})$ с ростом $d_{\mathrm{Al}}$ в области гелиевых температур, где $d_{\mathrm{Al}}$ - толщина $\mathrm{Al}$ слоя. Измерения сдвига частоты $\delta f$ диэлектрического резонатора СВЧ, находящегося в контакте с образцом, как функции температуры и $d_{\mathrm{Al}}$ показали, что (i) характер зависимости $\delta f(T)$ существенно зависит от $d_{\mathrm{Al}}$ и (ii) сдвиг резонансной частоты SN структур при температурах близких к критической температуре $T_{c}$ не описывается зависимостью вида const $/\left(1-T / T_{c}\right)$, которая типична для тонких сверхпроводящих пленок. Численные расчеты, выполненные в рамках модели Узаделя, хорошо описывают наблюдаемые эффекты. Таким образом, отмеченные аномалии электродинамических свойств SN структур можно объяснить наличием минищели в спектре квазичастиц, возникающей из-за эффекта близости в слое нормального металла, которая зависит от $d_{\mathrm{Al}}$, а также большой проводимостью $\mathrm{Al}$ слоя.
\end{abstract}

Ключевые слова: сверхпроводимость, эффект близости, микроволновый отклик, минищель.

DOI: 10.21883/FTT.2019.09.48117.14N

\section{1. Введение}

Измерение микроволнового импеданса $Z_{s}$ тонких сверхпроводящих пленок позволяет определить такой важный параметр сверхпроводника как глубина проникновения магнитного поля $\lambda$, а также исследовать ее зависимость от температуры (см., например, $[1,2]$ ). Данное обстоятельство связано с тем фактом, что обычно реальная часть импеданса сверхпроводника $R_{s}$ практически обращается в нуль уже при температуре немного ниже критической температуры сверхпроводящего перехода $T_{c}$, тогда как мнимая часть импеданса $X_{x}$ остается конечной и пропорциональной $\lambda^{2} / d$ (для пленок с толщиной $d \ll \lambda$ ). В настоящей работе мы теоретически и экспериментально исследуем микроволновой отклик гибридной структуры сверхпроводник $(S)$ - нормальный металл $(N)$, состоящей из грязного сверхпроводника $(\mathrm{MoN})$ с низкой статической проводимостью и нормального металла (Al) с высокой статической проводимостью. Наш интерес к такой задаче вызван недавней работой [3], в которой было обнаружено, что низкочастотная экранировка „грязной“ сверхпроводящей пленки может вырасти в несколько раз при нанесении на нее тонкого слоя низкоомного нормального металла. В этой работе было отмечено, что существенная зависимость экранировки от температуры для SN структур может возникать даже при низких температурах $\left(T \leq 0.5 T_{c}\right)$ в отличие от обычных сверхпроводящих пленок. Данный эффект был объяснен формированием наведенной сверхпроводимости в слое нормального металла за счет эффекта близости и большим отношением проводимостей $\sigma_{N} / \sigma_{S} \gg 1$. Отметим, что измерения эффективной глубины проникновения магнитного поля в работе [3] проводились с помощью индуктивного метода [4] с частотой переменного тока в передающей катушке $14 \mathrm{kHz}$.

Мы обнаружили, что электродинамический отклик подобной SN структуры на частоте $8 \mathrm{GHz}$ качественно отличен от отклика такой структуры на низких частотах, а также от высокочастотного отклика одиночной сверхпроводящей пленки. Мы показали, что мнимая часть импеданса SN структуры зависит от температуры немонотонным образом, при этом положение максимума $X_{s}$ зависит от толщины нормальной пленки $d_{N}$ : с ростом $d_{N}$ максимум сдвигается к меньшим температурам. Мы объясняем полученный результат нали- 
чием минищели $\varepsilon_{g}$ в спектре квазичастиц в пленке нормального металла, которая возникает из-за наличия эффекта близости. Хорошо известно, что величина минищели зависит от $d_{N}$ [5]. Это обстоятельство, а также условие $\sigma_{N} / \sigma_{S} \geq 1$ приводит к значительному вкладу нормальной части проводимости $\mathrm{SN}$ слоя в $X_{s}$ вплоть до температур $T<\varepsilon_{g}\left(d_{N}\right) / k_{B}$. Расчеты на основании микроскопической теории, выполненные в так называемом „грязном“ пределе, подтвердили данное объяснение, а также продемонстрировали, что в подобных структуpax возможно выполнение неравенства $R_{S}(T)>X_{S}(T)$ в широком диапазоне температур ниже $T_{c}$, которое не может быть реализовано для объемных сверхпроводников с обычной сверхпроводимостью [6]. Полученный нами результат говорит о принципиальной возможности оценки величины минищели гибридной SN структуры из измерения микроволнового импеданса. Обнаруженная сильная температурная зависимость $X_{S}(T)$ позволяет предложить использовать такие структуры в детекторах на кинетической индуктивности, работа которых основана на изменении кинетической индуктивности $L \sim X_{s}$ и соответствующей резонансной частоты LC контура при поглощении электромагнитного излучения [7].

\section{2. Экспериментальные результаты}

\section{1. Подготовка и характеризация образцов}

В работе исследована серия двухслойных тонкопленочных гибридных структур сверхпроводник $(S)$ нормальный металл $(N)$, выращенные методом магнетронного напыления с базовым уровнем вакуума порядка $1.5 \cdot 10^{-7}$ mbar на стандартных кремниевых подложках размером $10 \times 10 \mathrm{~mm}$ без удаления оксидного слоя. Сначала напылялся Мо в атмосфере смеси газов $\operatorname{Ar}: \mathrm{N}_{2}=10: 1$ при давлении $1 \cdot 10^{-3}$ mbar и комнатной температуре, что позволяло получить однородные сверхпроводящие MoN пленки с критической температурой порядка $8 \mathrm{~K}$. Затем in-situ напылялся Al в атмосфере аргона при давлением $1 \cdot 10^{-3} \mathrm{mbar}$. Толщины слоев были измерены методом рентгеновской дифрактометрии. Толщина сверхпроводящего слоя $d_{\mathrm{MoN}}$ для всех гибридных $S N$ образцов в серии составила $22 \mathrm{~nm}$, для образца сравнения (пленки MoN без покрытия нормальным металлом) $19 \mathrm{~nm}$, толщина слоя нормального металла $d_{\mathrm{Al}}$ варьировалась от 16 до $100 \mathrm{~nm}$.

Стандартные транспортные измерения на постоянном токе показали, что температурный коэффициент сопротивления $k \equiv R^{-1} d R / d T$ при $300 \mathrm{~K}$ изменяет знак и увеличивается по мере увеличения $d_{\mathrm{Al}}$. Такое поведение может быть связано с тем, что основным механизмом рассеяния в неупорядоченном слое $\mathrm{MoN}$ является электрон-электронное рассеяние, что приводит к отрицательным значениям $k$. Покрытие Al с большой проводимостью шунтирует плохо проводящий слой $\mathrm{MoN}$, в результате сопротивление $S N$ структуры все более приближается к сопротивлению нормального металла с положительным $k$ из-за преобладающего электронфононного механизма рассеяния. Удельное сопротивление при комнатной температуре $\mathrm{MoN}$ составило $98 \mu \Omega \cdot \mathrm{cm}$. Удельное сопротивление $\mathrm{Al}$ при комнатной температуре составило: $7.1 \mu \Omega \cdot \mathrm{cm}$ для пленки $16 \mathrm{~nm}$, $4.3 \mu \Omega \cdot \mathrm{cm}$ для пленки $35 \mathrm{~nm}$ и $3.3 \mu \Omega \cdot \mathrm{cm}$ для пленки $60 \mathrm{~nm}$.

Исследование особенностей экранирующих свойств тонкопленочных SN структур осуществлялось двумя методами:

(i) путем измерения коэффициента взаимной индукции двух катушек с образцом между нами $[4,8]$;

(ii) путем измерения сдвига резонансной частоты диэлектрического резонатора, находящегося в контакте с образцом [9].

\section{2. Измерение коэффициента взаимной индукции}

Для исследования магнитной экранировки на низких частотах образец помещался между двумя аксиальносимметричными индуктивно связанными катушками. Переменный ток с частотой $14 \mathrm{kHz}$, проходящий через возбуждающую катушку, порождает переменное магнитное поле, которое возбуждает ток в приемной катушке. При переходе образца в сверхпроводящее состояние магнитное поле частично экранируется и потому коэффициент взаимной индукции, который определяется как отношение амплитуды переменного тока в приемной катушке к амплитуде возбуждающего тока, уменьшается. Как было показано в работах $[4,10]$, для тонких пленок с толщиной $d$ справедливо соотношение

$$
M \simeq C \frac{\lambda}{\sinh (d / \lambda)} \propto \frac{\lambda^{2}}{d}=\Lambda \text { при } d \ll \lambda,
$$

где $C$ - геометрический коэффициент, $\lambda-$ лондоновская глубина проникновения, $\Lambda \equiv \lambda^{2} / d-$ так называемая эффективная (или пирловская) глубина магнитного поля. Отметим, что соотношение (1) справедливо, если, во-первых, выполнено соотношение $\Lambda \ll D \ll L$, где $D$ - параметр, по порядку величины равный расстоянию между катушками или диаметру катушек, $L-$ характерный латеральный размер пленки. Для SN структуры конечной толщины следует учитывать неоднородность токов по толщине структуры [из-за зависимости $\left.1 / \lambda^{2}(z)\right]$, поэтому для описания степени эффективности экранировки можно ввести параметр

$$
\frac{1}{\Lambda}=\int_{0}^{d_{S}+d_{N}} \frac{d z}{\lambda^{2}(z)}, \text { тогда } M \propto \Lambda .
$$

Здесь $d_{S}$ и $d_{N}$ - толщины сверхпроводящего и нормального слоев соответственно; $\lambda(z)$ - локальная лондоновская глубина проникновения магнитного поля, которую можно вычислить в рамках микроскопической теории [3]. 

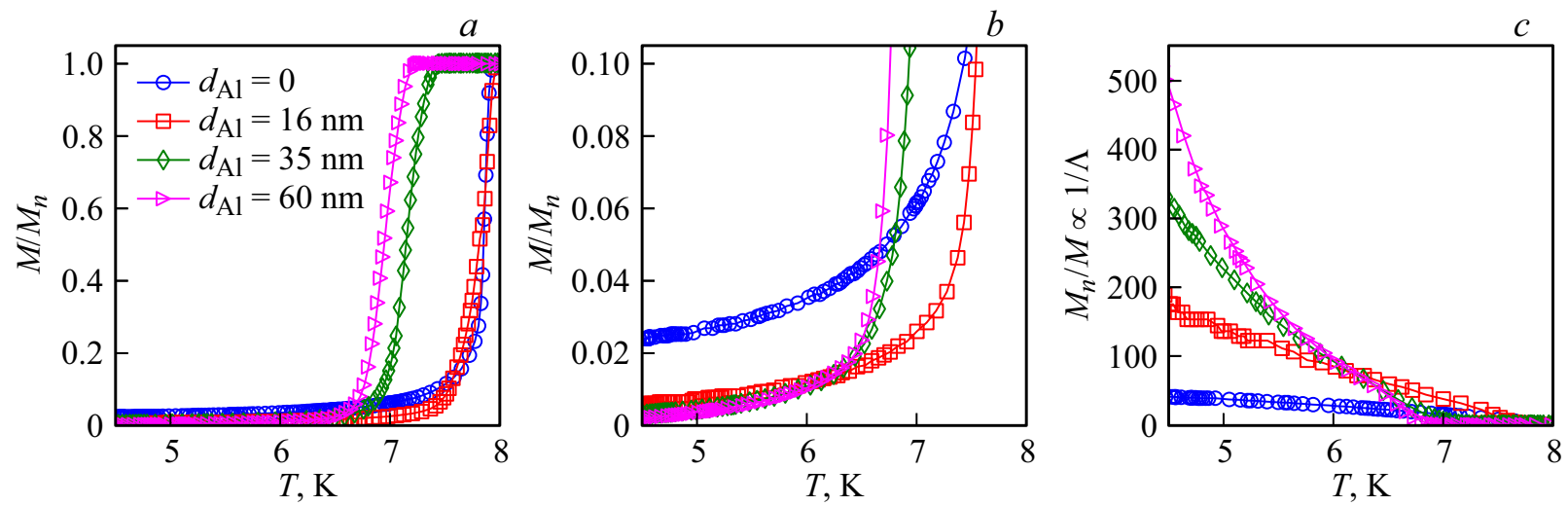

Рис. 1. (цветной онлайн) $(a, b)$ Зависимость нормированного коэффициента взаимоиндукции $M / M_{n}$ от температуры для $\mathrm{SN}$ структур с различной толщиной $\mathrm{Al}$ слоя: $d_{\mathrm{MoN}}=16 \mathrm{~nm}$ и $d_{\mathrm{Al}}=0, d_{\mathrm{MoN}}=20 \mathrm{~nm}$ и $d_{\mathrm{Al}}=16 \mathrm{~nm}, d_{\mathrm{MoN}}=20 \mathrm{~nm}$ и $d_{\mathrm{Al}}=35 \mathrm{~nm}$, $d_{\mathrm{MoN}}=20 \mathrm{~nm}$ и $d_{\mathrm{Al}}=60 \mathrm{~nm} ; M_{n}$ - величина коэффициента взаимной индукции при $T>T_{c}$. (c) Зависимость $M_{n} / M \propto 1 / \Lambda$ от температуры тех же образцов. На $(b)$ изображены в увеличенном масштабе зависимости $M / M_{n}$ от $T$, показанные на рис. $1, a$.

На рис. $1, a, b$ показаны типичные зависимости нормированного коэффициента взаимной индукции от температуры для SN структур с различной толщиной $\mathrm{Al}$ слоя. Легко видеть, что по мере увеличения $d_{\mathrm{Al}}$ происходит уменьшение критической температуры, что также подтверждается транспортными измерениями. На рис. 1, $c$ показана температурная зависимость обратного коэффициента взаимной индукции для тех же образцов. Эти результаты свидетельствуют об увеличении плотности сверхпроводящей фракции в SN структурах и, соответственно, об уменьшении глубины проникновения магнитного поля при гелиевых температурах по мере увеличения $d_{\mathrm{Al}}$ по сравнению с пленкой $\mathrm{MoN}$ [3].

\section{3. Резонаторная СВЧ-спектроскопия}

Для исследования особенностей высокочастотных свойств тонкопленочных SN структур были проведены измерения частотной зависимости коэффициента отражения электромагнитной волны (спектр отражения) от антенны, которая индуктивно связана с диэлектрическим резонатором. Диэлектрический резонатор изготовлен из рутила $\left(\mathrm{TiO}_{2}\right)$, имеет форму цилиндра диаметром $4 \mathrm{~mm}$ и высотой $2 \mathrm{~mm}$ и обладает высокой диэлектрической проницаемостью при гелиевых температурах $(\varepsilon>100)$, что приводит к тому, что электромагнитное поле в основном локализуется внутри резонатора [9]. При исследовании частотной зависимости коэффициента отражении одна из стенок резонатора приводилась в контакт с поверхностью исследуемого образца, поэтому реактивные свойства сверхпроводящегообразца, обусловленные его кинетической индуктивностью, меняли эквивалентную индуктивность системы „резонатор-образец“.

Для измерений была выбрана мода $T E_{011}$, в которой электрическое поле содержит только азимутальные компоненты вдоль плоской границы „резонатор-образец“ (рис. 2,a). Размеры резонатора выбраны так, что рабочая частота находится в окрестности $8 \mathrm{GHz}$. Возбуждение резонатора осуществлялось круглой петлевой антенной, диаметр которой совпадал с диаметром резонатора. Расстояние между антенной и резонатором выбиралась так, чтобы выполнялось условие слабой связи с резонатором. Мощность непрерывного источника СВЧизлучения составляла $1 \mathrm{~mW}$, что обеспечивало линейный режим отражения от образца во всем диапазоне частот и рабочих температур.

На рис. 2, $b$ приведена эквивалентная схема резонатора, связанного с передающей линией. Диэлектрический резонатор вместе с окружением можно описать как резонансный $L_{0}-C_{0}-R_{0}$ контур. Антенну будем рассматривать как индуктивный элемент $L^{\prime}$, индуктивно связанный с резонатором, с коэффициентом взаимной индукции $M$. Эффективный импеданс системы „антеннарезонатор“ можно записать как

$$
\hat{Z}_{\mathrm{eff}}=-i \omega L^{\prime}+i \frac{\omega^{2} M^{2}}{\omega L_{0}+i R_{0}-\left(\omega C_{0}\right)^{-1}} .
$$

Диапазон частот, в котором проводятся измерения, мал по сравнению с собственной частотой колебательного контура: $\delta \omega=\omega-\omega_{0} \ll \omega_{0}$. Для упрощения выражения (3) введем следующие параметры: собственную частоту ненагруженного резонатора $\omega_{0}^{2}=1 / L_{0} C_{0}$, коэффициент затухания $\alpha=R_{0} / 2 L_{0}$, коэффициент связи $\Gamma=\omega_{0} M^{2} /\left(2 Z_{\omega} L_{0}\right)$; а также $\Gamma^{\prime}=\omega_{0} L^{\prime} / Z_{\omega}$

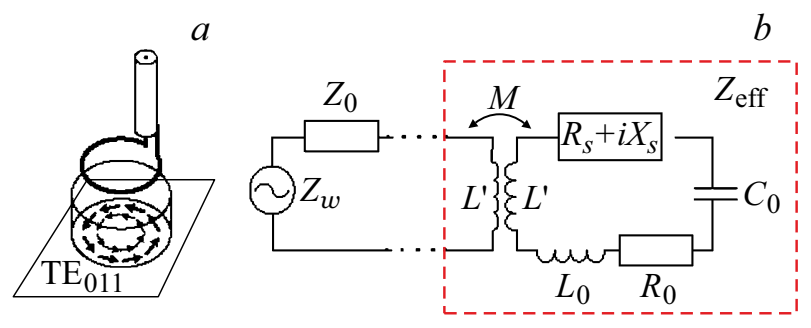

Рис. 2. Структурная схема резонатора и эквивалентная схема. 
и $\gamma=\Gamma /\left(1+\Gamma^{\prime 2}\right)$, где $Z_{\omega}=50 \Omega$ есть волновой импеданс линии передач. В этом случае эффективный импеданс системы „резонатор-образец“ (3) равен

$$
Z_{\mathrm{eff}} \simeq-i Z_{\omega} \Gamma^{\prime}+i \frac{Z_{\omega} \Gamma}{\delta \omega+i \alpha}
$$

Если в линии передач отсутствуют неоднородности, обуславливающие формирование стоячих волн в передающем тракте, коэффициент отражения по амплитуде $r$ связан с $Z_{\text {eff }}$ выражением [11]

$$
r=\frac{Z_{\omega}-Z_{\mathrm{eff}}}{Z_{\omega}+Z_{\mathrm{eff}}}
$$

После подстановки (4) в (5) получаем выражение для коэффициента отражения по мощности

$$
r^{2}=1-\frac{4 \alpha \gamma}{\left(\delta \omega-\gamma \Gamma^{\prime}\right)^{2}+(\alpha+\gamma)^{2}} .
$$

Таким образом, коэффициент отражения $|r|^{2}$ как функция $\delta \omega$ описывается функцией Лоренца, при этом минимальное значение соответствует частоте $\omega^{*}=\omega_{0}+\gamma \Gamma^{\prime}$, которая отличается от собственной частоты $\omega_{0}$ в меру малости коэффициента связи.

Будем считать, что влияние поверхностного импеданса $Z_{s}=R_{s}+i X_{s}$ на электродинамические свойства $\mathrm{SN}$ структур может быть учтено включением импеданса $Z_{s}$ последовательно с $L_{0}-C_{0}-R_{0}$ контуром, описывающим резонатором. Это эквивалентно изменению полной индуктивности $\tilde{L} \simeq L_{0}+X_{s}(\omega) / \omega$ и полного сопротивления $\tilde{R}=R_{0}+R_{s}(\omega)$ резонатора. При условии $X_{s} / \omega \ll L_{0}$ коэффициент отражения попрежнему будет описываться функцией Лоренца, однако положение минимума коэффициента отражения будет соответствовать частоте

$$
\begin{aligned}
\omega_{\min } & =2 \pi f_{\min } \simeq\left(L_{0} C_{0}+\frac{X_{s}\left(\omega_{0}\right)}{\omega_{0}} C_{0}\right)^{-1 / 2}+\gamma \Gamma^{\prime} \\
& \simeq \omega^{*}-\frac{\omega_{0}}{2 L_{0}} \frac{X_{s}\left(\omega_{0}\right)}{\omega_{0}}
\end{aligned}
$$

Измерения выполнялись на частоте вблизи $8 \mathrm{GHz}$ в окне шириной до $300 \mathrm{MHz}$. Для каждого образца были получены частотные зависимости коэффициента отражения $|r|^{2}$ для температур от $4.2 \mathrm{~K}$ до $8 \mathrm{~K}$. Спектры отражения для всех температур были аппроксимированы функцией Лоренца, что позволило оценить положение минимума и получить зависимость его положения от температуры. Заметим, что диэлектрическая проницаемость резонатора и $\omega^{*}$ практически не зависит от температуры при измерениях в температурном интервале от 4 до $20 \mathrm{~K}$, поэтому мы можем легко отделить температурно-зависящий вклад, пропорциональный $X_{s}$, от остальных слагаемых.

На рис. 3 приведены графики температурных зависимостей частоты, соответствующей минимуму коэффициента отражения для линий передач с резонатором,
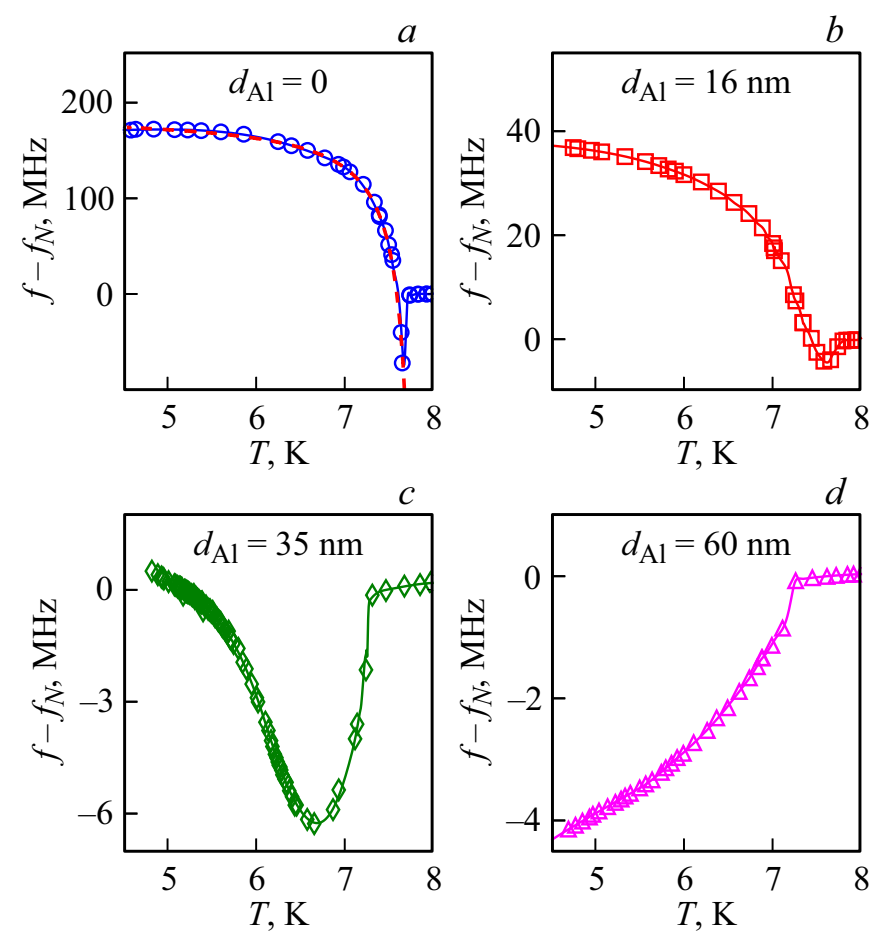

Рис. 3. (цветной онлайн) Температурные зависимости нормированного сдвига резонансной частоты диэлектрического резонатора, одна из стенок которого находится в контакте с исследуемой тонкопленочной гибридной SN структурой: (a) $\quad d_{\mathrm{MoN}}=19 \mathrm{~nm} \quad$ и $\quad d_{\mathrm{Al}}=0 ; \quad(b) \quad d_{\mathrm{MoN}}=22 \mathrm{~nm} \quad$ и $d_{\mathrm{Al}}=16 \mathrm{~nm} ;(c) d_{\mathrm{MoN}}=22 \mathrm{~nm}$ и $d_{\mathrm{Al}}=35 \mathrm{~nm},(d) d_{\mathrm{MoN}}=22 \mathrm{~nm}$ и $d_{\mathrm{Al}}=60 \mathrm{~nm}$. На рис. (a) пунктирной линией показан график зависимости вида $f=A+B /\left(1-T / T_{c}\right)$, где подгоночные параметры $A, B$ и $T_{c}$ были определены по методу наименьших квадратов.

находящегося в контакте с пленкой $\mathrm{MoN}(a)$ и планарными гибридными структурами $\mathrm{MoN} / \mathrm{Al}$ с различной толщиной $\mathrm{Al}$ слоя $(b-d)$.

Было обнаружено, что при нагреве образца сверхпроводящей MoN пленки от гелиевых температур резонансная частота монотонно смещается в область меньших значений (за исключением узкой области вблизи $T_{c}$ ) и затем выходит на постоянное значение (рис. 3, $a$ ). Такое поведение является типичным для одиночных сверхпроводящих пленок, при этом уменьшение $f_{\min } \mathrm{c}$ ростом $T$ свидетельствует об увеличении кинетической индуктивности образца, обусловленной сверхпроводящими электронами [12]. Экспериментальные зависимости $f_{\min }(T)$ хорошо описываются модельной функцией вида $f_{\text {appr }}(T)=A+B /\left(1-T / T_{c}\right)$, где постоянная $A$ зависит от частоты выбранной моды и постоянная $B$ зависит от абсолютного значения лондоновской глубины проникновения, толщины образца и коэффициента связи. Заметим, что именно такая температурная зависимость соответствует зависимости $\Lambda(T)=\lambda^{2} / d_{S}(\lambda-$ лондоновская глубина проникновения в сверхпроводник). Отклонение наблюдаемой зависимости $f_{\min }(T)$ от 
аппроксимирующей зависимости $f_{\text {appr }}(T)$ наблюдается вблизи $T_{c}$, где, по всей видимости, нельзя пренебрегать активной частью проводимости по сравнению с реактивной частью.

Зависимости $f_{\min }(T)$ для $\mathrm{MoN} / \mathrm{Al}$ структур (рис. $3, b, c)$ существенно отличаются от типичных зависимостей для сверхпроводящих пленок: сдвиг частоты становится немонотонной функцией температуры и участок на зависимостях $f_{\min }(T)$, где резонансная частота увеличивается, выражен тем сильнее, чем больше $d_{\mathrm{Al}}$. Для $\mathrm{MoN} / \mathrm{Al}$ структуры с $d_{\mathrm{Al}}=60 \mathrm{~nm}$ (рис. $3, d$ ) в доступном нам для измерений температурном диапазоне резонансная частота монотонно увеличивается. Обсуждение полученных результатов и их сравнение с теорией приводится в следующем разделе.

\section{3. Поверхностный импеданс для тонкопленочных SN структур: теория и обсуждение эксперимента}

Расчет импеданса тонкопленочной SN структуры был выполнен в рамках микроскопических модели в так называемом „грязном“ пределе с использованием уравнений Узаделя.

Введем импеданс $Z_{s}$ нашей структуры как отношение амплитуды тангенциальной электрического поля $E_{\|}(0)$ к амплитуде полного тока $I$, пересекающего площадку единичной ширины, расположенную перпендикулярно току [13]:

$$
Z_{s} \equiv \frac{E_{\|}(0)}{\int_{z_{1}}^{z_{2}} j_{\|}(z) d z},
$$

где $j_{\|}$есть плотность электрического тока, текущего параллельно поверхности, ось $z$ выбрана перпендикулярно поверхности структуры, интегрирование выполняется по всем доступным значениям координаты $z_{1} \leq z \leq z_{2}$ с ненулевой плотностью тока. Предполагая, что справедливо локальное соотношение $j_{\|}(z)=\sigma(z) E_{\|}(0)$, получаем

$$
Z_{s}=\left(\int_{0}^{d} \sigma(z) d z\right)^{-1},
$$

где $\sigma=\sigma_{1}+i \sigma_{2}-$ комплексная проводимость [12], $d=d_{S}+d_{N}-$ толщина $\mathrm{SN}$ бислоя. Для микроскопического расчета $\sigma(z)$ мы использовали численное решение уравнения Узаделя для аномальной $F(z)$ и нормальной $G(z)$ функций Грина в „грязном“ пределе, а также уравнения самосогласования для сверхпроводящего параметра порядка (подробно базовые уравнения и граничные условия описаны в работе [3]).

Связь локальной проводимости $\sigma(z)$ и функций Грина $F(z)$ и $G(z)$ приведена в работе [14] (соотношения (В14) и (В16)), которую удобно использовать для численного анализа. При моделировании мы вычислили $Z_{s}$ на нулевой частоте (см. рис. 4,5), поскольку
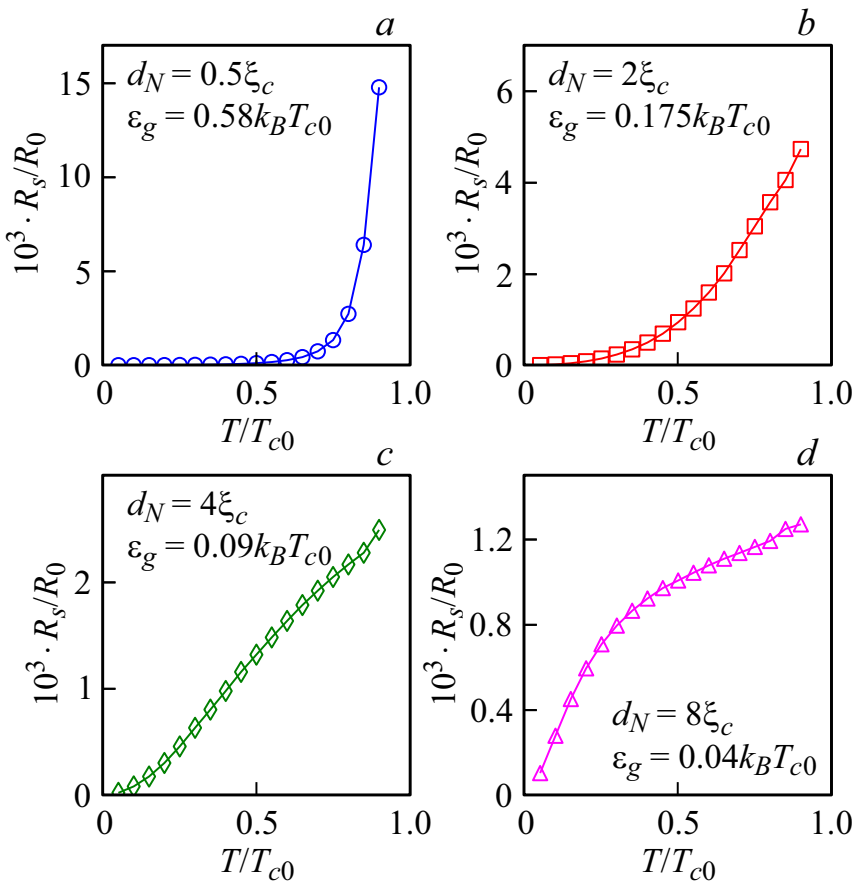

Рис. 4. Температурная зависимость действительной части импеданса SN структуры для частоты $8.5 \mathrm{GHz}$, фиксированной толщине сверхпроводящего слоя $d_{S}=3 \xi_{c}$ и разных толщинах нормального слоя: $(a) d_{N}=0.5 \xi_{c},(b) d_{N}=2 \xi_{c},(c) d_{N}=6 \xi_{c}$, (d) $d_{N}=10 \xi_{c} ; \xi_{c}=\left(\hbar D_{S} / k_{B} T_{c 0}\right)^{1 / 2}, \sigma_{N} / \sigma_{S}=10, R_{0}=1 /\left(\sigma_{S} \xi_{c}\right)$. Величина рассчитанной минищели $\varepsilon_{g}$ при $T=0.1 T_{c 0}$ для различных $d_{N}$ приведена на рисунке.
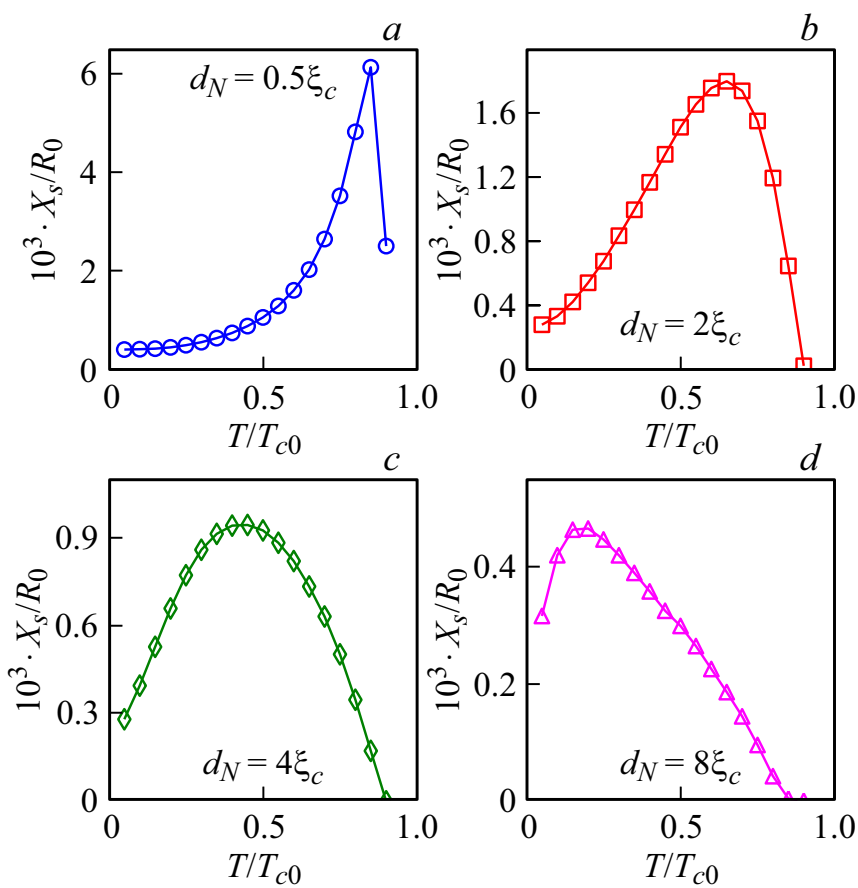

Рис. 5. Температурная зависимость мнимой части импеданса SN структуры для частоты $8.5 \mathrm{GHz}$, фиксированной толщине сверхпроводящего слоя $d_{S}=3 \xi_{c}$ и разных толщинах нормального слоя: (a) $d_{N}=0.5 \xi_{c}$, (b) $d_{N}=2 \xi_{c},(c) d_{N}=6 \xi_{c}$, (d) $d_{N}=10 \xi_{c} ; \xi_{c}=\left(\hbar D_{S} / k_{B} T_{c 0}\right)^{1 / 2}, \sigma_{N} / \sigma_{S}=10, R_{0}=1 /\left(\sigma_{S} \xi_{c}\right)$. 


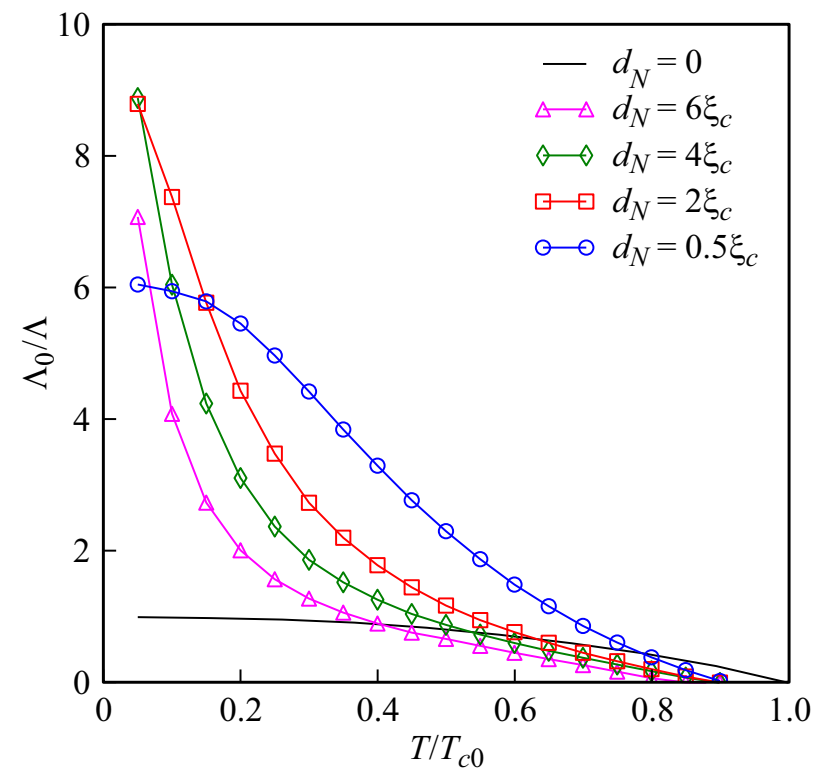

Рис. 6. Температурная зависимость обратной эффективной глубины проникновения магнитного поля $\Lambda^{-1}$ $\left(\Lambda=\int \sigma_{2}(z) \omega \mu_{0} d z=\int d z / \lambda(z)^{2}, \Lambda_{0}=d_{S} / \lambda(0)^{2}, \lambda(0)-\right.$ лондоновская глубина проникновения магнитного поля одиночной сверхпроводящей пленки) рассчитанная в рамках модели Узаделя для $\mathrm{SN}$ бислоя с разными $d_{N}$. Толщина $\mathrm{S}$ слоя одинакова и равна $3 \xi_{c}$.

используемая в эксперименте частота много меньше как $k_{B} T_{c}$, так и $k_{B} T$ (при использованных в эксперименте температурах).

Эволюция $X(s)$ с ростом $d_{N}$ (см. рис. 5) качественно совпадает с результатами эксперимента. Полученные результаты можно объяснить как наличием минищели $\varepsilon_{g}$ в слое нормального металла, которая зависит от $d_{N}$, так и большим отношением $\sigma_{S} / \sigma_{N} \gg 1$. Действительно, для $\mathrm{SN}$ структуры конечной толщины $X_{s}$ можно записать следующим образом:

$$
X_{s}=\frac{G_{2}}{G_{1}^{2}+G_{2}^{2}},
$$

где $G_{1,2}=\int_{0}^{d_{S}+d_{N}} \sigma_{1,2}(z) d z$ Поскольку $\sigma_{S} \ll \sigma_{N}$, то основной вклад в $G_{1}$ дает нормальный слой и $G_{1} \simeq \sigma_{N} d_{N}$ до температур больших $T_{g}=\varepsilon_{g}\left(d_{N}\right) / k_{B}$. Данный результат можно легко получить и без численного расчета. Действительно, согласно уравнению (11) из $[15] \sigma_{1}$ выражается через интеграл по энергии, где подинтегральное выражение представляет из себя произведение производной функции распределения по энергии $\partial \tanh \left(E / 2 k_{B} T\right) / \partial E$ (при низких частотах $\omega \ll T$ ) и спектральной функции, не равной нулю при $E>\varepsilon_{g}$. Так как $\partial \tanh \left(E / 2 k_{B} T\right) / \partial E \rightarrow 0$ при $E>k_{B} T$, то при низких температурах $\left(T<\varepsilon_{g} / k_{B}\right)$ реальная часть проводимости экспоненциально падает с уменьшением температуры.

С другой стороны, в пренебрежении наведенной сверхпроводимостью в нормальном слое и со- ответствующим увеличением экранирующего отклика можно записать, что $G_{2}=d_{S} \sigma_{S} \pi \Delta(0) /(\hbar \omega f(T))$ где $\Delta(0)=1.76 k_{B} T_{c 0}$, а $f(T)=\lambda(T)^{2} / \lambda(0)^{2}$. Подставив эти выражения в уравнение (9) нетрудно убедиться, что из-за $\sigma_{N} / \sigma_{N} \gg 1$ температура, при которой $G_{1} \simeq G_{2}$ и $X_{S}(T)$ достигает максимального значения, находится не вблизи $T_{c}$, как в обычных сверхпроводниках, а при более низких температурах (при этом максимум сдвигается в меньшие температуры с ростом $d_{N}$ ). Данный эффект практически незаметен для низких частот изза большого отношения $k_{B} T_{c} / \hbar \omega \gg 1$, что и приводит к качественно иному поведению $X_{s} \sim \Lambda$. в наших низкочастотных индуктивных измерениях (рассчитанная $\Lambda(T)$ для различных толщин $\mathrm{N}$ слоя представлена на рис. 6).

Отметим, что использованная модель дает качественное объяснение полученных экспериментальных результатов, однако количественного согласия добиться не получается. Так, согласно теоретическим расчеам резкий рост $1 / \Lambda$ должен происходить при достаточно низких температурах, с последующим выходом на насыщение при температуре $T<\varepsilon_{g} / k_{B}$, и чем больше толщина нормального слоя, тем меньше должна быть эта температура (что связано с зависимостью минищели от $d_{N}$ ) см. рис. 6. В эксперименте такой рост происходит при более высоких температурах (насыщения мы не видим, так как оно должно происходить при более низких температурах). Тем не менее, видно (см. рис. 1, $c$ ), что с увеличением толщины нормального слоя пересечение зависимостей $1 / \Lambda(T)$, соответствующих разным $d_{N}$, происходит при все более низких температурах, что качественно согласуется с теорией. В работе [3] было предположено, что количественное рассогласование между теорий и экспериментом может быть связано с тем, что длина свободного пробега электронов в высокоомных сверхпроводниках (с малой $\sigma$ ) оказывается порядка фермиевской длины волны $\lambda_{F}$ что ставит вопрос о количественной применимости уравнений Узаделя в таких материалах. Введение конечной прозрачности границы между $S$ и $N$ слоем не позволит решить этот вопрос, так как это будет приводить к уменьшению эффекта близости, тогда как экспериментальные результаты указывают на более сильный эффект близости (более сильное уменьшение $\Lambda$ ) чем предсказывает модель Узаделя с абсолютно прозрачной границей. По этой причине мы также не проводим количественное сравнение рассчитанного и экспериментального импедансов.

Результаты моделирования показывают, что наличие низкоомного нормального слоя приводит к выполнению условия $R_{s}>X_{S}$ в широком диапазоне температур, тогда как в обычной сверхпроводящей пленке это соотношение выполняется только в малой окрестности температур ниже $T_{c}$. Заметим, что в случае толстых сверхпроводящих пленок (с толщиной много большей скинслоя) $R_{s} \simeq X_{s}$ при всех температурах, за исключением случая наличия необычной спин-триплетной нечетной по времени сверхпроводимости, где возможно обратное отношение $R_{s}>X_{s}[15]$. 
Отметим, что наши результаты для зависимости $X_{S}(T)$ и ее изменение с ростом толщины $\mathrm{Al}$ качественно отличаются от результатов, обнаруженных для $\mathrm{Nb} / \mathrm{Al}$ и $\mathrm{Nb} / \mathrm{Cu}$ бислоев [16,17]. По-видимому отличие связано как с небольшим отношением проводимостей в этих парах металлов, так и с большой толщиной $\mathrm{Nb}$ слоя, значительно превышающей как $\lambda(0)$ ниобия, так и толщины слоев нормальных металлов.

Конечное значение $R_{s}$ должно приводить к низкой добротности резонаторов на основе SN бислоев (за исключением области температур $T \ll T_{g}$ ), что было обнаружено в эксперименте на $\mathrm{NbTiN} / \mathrm{Au}$ гибридной структуре [18]. Однако сильная температурная зависимость $X_{s}(T)$ вместе с достаточно низким $R_{s}$ при низких температурах в случае малой толщины нормального слоя позволяет предложить такие структуры в качестве активного элемента в детекторах электромагнитного излучения на кинетической индуктивности. Действительно, работа таких детекторов основана на разогреве сверхпроводника в результате поглощения излучения и соответствующего изменения кинетической индуктивности $L \sim X_{s}$. Это изменение $L$ можно установить по изменению резонансной частоты соответствующего резонансного контура [7]. Очевидно, что чем сильнее температурная зависимость $X_{s}(T)$, тем выше должна быть чувствительность такого детектора.

\section{4. Заключение}

Мы показали, что низкочастотные свойства гибридных сверхпроводящих систем с большим отношением проводимостей (таких как планарные $S N$ структуры) могут существенно отличаться от высокочастотных свойств обычного низкотемпературного сверхпроводника в широком интервале температур. Нами была изготовлена серия тонкопленочных гибридных структур сверхпроводник $(\mathrm{MoN})$ - нормальный металл $(\mathrm{Al})$, которые характеризуются, во-первых, эффектом близости между слоями и, во-вторых, большой разностью проводимостей слоев в нормальном состоянии. Низкочастотные измерения магнитной экранировки с помощью двух индуктивно-связанных катушек показали, что наличие Al покрытия приводит к уменьшению глубины проникновения магнитного поля при низких температурах по мере увеличения толщины $\mathrm{Al}$ слоя до толщин порядка 50-100 nm. СВЧ-измерения смещения резонансной частоты $f_{\min }$ диэлектрического резонатора, одно из оснований которого находилось в контакте со сверхпроводящим образцом, как функции температуры показали, что характер зависимости $f_{\min }(T)$ существенно зависит от толщины Al слоя. Экспериментальные результаты по микроволновому отклику качественно хорошо объясняются наличием наведенной минищели в нормальном металле и большим отношением нормальных проводимостей $\mathrm{MoN}$ и $\mathrm{Al}$ подтверждаются расчетами, выполненными в рамках модели Узаделя.

\section{Финансирование работы}

В работе использовано оборудование Центра коллективного пользования „Физика и технология микро- и наноструктур“ ИФМ РАН.

Работа выполнена при поддержке РФФИ (проект 19-02-00528, численное моделирование) и РНФ (проект 15-12-10020, экспериментальная работа: изготовление образцов и проведение измерений).

\section{Список литературы}

[1] S. Sridhar. J. Appl. Phys. 63, 15 (1988).

[2] М.Р. Трунин. УФН 168, 931 (1998).

[3] D.Yu. Vodolazov, A.Yu. Aladyshkin, E.E. Pestov, S.N. Vdovichev, S.S. Ustavshikov, M.Yu. Levichev, A.V. Putilov, P.A. Yunin, A.I. El'kina, N.N. Bukharov, A.M. Klushin. Supercond. Sci. Technol. 31, 115004 (2018).

[4] J.H. Claassen, M.L. Wilson, J.M. Byers, S. Adrian. J. Appl. Phys. 82, 3028 (1997).

[5] W. Belzig, C. Bruder, G. Schon. Phys. Rev. B 549443 (1996).

[6] M. Tinkham. Introduction to Superconductivity, McGrawHill, N. Y. (1996).

[7] P.K. Day, H.G. LeDuc, B.A. Mazin, A. Vayonakis, J. Zmuidzinas. Nature 425, 817 (2003).

[8] A.T. Fiory, A.F. Hebard, P.M. Mankiewich, R.E. Howard. Appl. Phys. Lett. 52, 2165 (1988).

[9] K. Torokhtii, C. Attanasio, C. Cirillo, E.A. Ilyina, N. Pompeo, S. Sarti, E. Silva. Physica C 479, 140 (2011).

[10] J.R. Clem, M.W. Coffey. Phys. Rev. B 46, 14662 (1992).

[11] A.M. Portis, D.W. Cooke, E.R. Gray. J. Supercond. 3, 297 (1990).

[12] J.I. Gittleman, B. Rosenblum. Proc. IEEE 52, 1138 (1964).

[13] А.А. Абрикосов. Основы теории металлов. Наука, М. (1987). $520 \mathrm{c}$.

[14] Ya.V. Fominov, M. Houzet, L.I. Glazman. Phys. Rev. B 84, 224517 (2011).

[15] S.V. Bakurskiy, Ya.V. Fominov, A.F. Shevchun, Y. Asano, Y. Tanaka, M.Yu. Kupriyanov, A.A. Golubov, M.R. Trunin, H. Kashiwaya, S. Kashiwaya, Y. Maeno. Phys. Rev. B 98, 134508 (2018).

[16] M.S. Pambianchi, S.N. Mao, S.M. Anlage. Phys. Rev. B 52, 4477 (1995).

[17] M.S. Pambianchi, L. Chen, S.M. Anlage. Phys. Rev. B 54, 3502 (1996).

[18] R. Barends, W.K.-G. Daalman, A. Endo, S. Zhu, T. Zijlstra, T.M. Klapwijk. AIP Conf. Proc. 1185, 152 (2009).

Редактор Ю.Э. Китаев 